TENDENCIAS

Revista de la Facultad de Ciencias

Económicas y Administrativas.

Universidad de Nariño

Vol. XVIII. No. 1 - 1er. Semestre 2017,

Enero-Junio - Páginas 85-100

\title{
FACTORES CLAVES DE ÉXITO EN LA IMPLEMENTACIÓN DE LEAN MANUFACTURING EN ALGUNAS EMPRESAS CON SEDE EN COLOMBIA
}

\author{
SUCCESS KEY FACTORS ON LEAN MANUFACTURING IMPLEMENTATION, AT SOME \\ COMPANIES BASED IN COLOMBIA
}

\section{FATORES CHAVE DE SUCESSO NA IMPLEMENTAÇÃO DE FABRICAÇÃO LEAN, EM ALGUMAS EMPRESAS SEDE NA COLOMBIA}

\author{
Por: LEÓN - Gonzalo Emilio, MARULANDA - Natalia, GONZÁLEZ - Henry Helí
}

\begin{abstract}
Magister en Negocios Internacionales Institución Universitaria Esumer, Docente de Mercadeo, Institución Universitaria ESCOLME, Medellín, gonzalo.leon@visionglobal.com.co, Colombia. Magister en Ingeniería Industrial Universidad Nacional de Colombia, Docente de Logística Corporación Universitaria Minuto de Dios sede Bello, natalia.marulanda@unimunuto.edu, Colombia Magister en Educación Universidad de Manizales, Docente de Logística Corporación Universitaria Minuto de Dios sede Bello, henry.gonzalez@uniminuto.edu, Colombia.
\end{abstract}

\section{RESUMEN}

La investigación del presente documento pretendió identificar en cinco empresas de diferentes sectores de la economía, en Colombia, cuáles han sido los factores claves que han permitido implementar con éxito herramientas de Manufactura Esbelta o Lean Manufacturing. Estas herramientas surgen de la filosofía japonesa, que pretende hacer más con menos, eliminando todo tipo de desperdicios en materiales y recursos; mediante la aplicación de diferentes herramientas administrativas y de producción que incluye entre otras, la mejora continua. Se entiende por desperdicio, todas aquellas actividades que no aportan valor al producto y por las cuales el cliente no está dispuesto a pagar. Se buscó identificar también cuales han sido sus principales logros y barreras en la implementación, a partir de una investigación de aspectos cualitativos mediante la metodología de estudio de caso. Se encontró que el compromiso de los dueños y directivos, el liderazgo visto desde las competencias Lean, el cuidadoso diseño de indicadores y su seguimiento y el entrenamiento en la filosofía y operación, son esenciales a la hora de realizar la implementación; estos ayudaron en la mejora de aspectos relevantes como la productividad y rentabilidad. En algunas, la implementación exitosa, permitió extender su aplicación a sus proveedores más importantes. 
Palabras clave: Compromiso de la dirección, Entrenamiento Lean, Implementación, Lean Manufacturing, Mejora Continua, Liderazgo,

JEL: L23, O33

\begin{abstract}
This research intended to identify in five companies of different sectors of the economy and based in Colombia, which has been the key factors that have allowed to successfully implement tools of Lean Manufacturing. These tools came from Japanese philosophy that aims to do more with less, eliminating all types of waste in materials and resources; by applying different administrative and production tools including among others, continuous improvement. It means waste, all activities that do not add value to the product and for which the customer is not willing to pay. We sought to identify also which have been their main achievements and barriers in this process, from the research of qualitative aspects, through case study methodology. Found that the commitment of the owners and managers, leadership, seen from Lean skills; careful design of indicators and their monitoring and the training in philosophy and operation, are essential when it comes to implementation, it improves aspects as important as productivity and profitability. In some of them the successful implementation allowed to extend its application to their main suppliers.
\end{abstract}

Keywords: Lean manufacturing, Implementation, Continuous Improvement, Leadership, Lean Training, Owners and management Commitment.

JEL: L23, O33

\title{
RESUMO
}

Esta pesquisa tem como objetivo identificar em cinco empresas de diferentes setores da economia e com base na Colômbia, que têm sido os principais fatores que permitiram a implementar ferramentas manufatura enxuta ou Lean Manufacturing. Estas ferramentas vieram de filosofia japonesa que visa a fazer mais com menos, eliminando todos os tipos de resíduos em materiais e recursos; através da aplicação de diferentes ferramentas administrativas e de produção, incluindo, entre outros, a melhoria contínua. Isso significa desperdício, todas as atividades que não agregam valor ao produto e para o qual o cliente não está disposto a pagar Ele também procurou identificar quais foram as suas principais realizações e obstáculos neste processo, a partir da investigação dos aspectos qualitativos, através de estudos de caso. Descobriram que o compromisso dos proprietários e gerentes, liderança, visto de habilidades de Lean; design cuidadoso dos indicadores e sua monitorização e da formação em filosofia e operação, são essenciais quando se trata de execução, melhorando aspectos tão importantes como a produtividade e rentabilidade. Em alguns deles a implementação bem-sucedida permissão para estender a sua aplicação aos seus principais fornecedores.

Palavras-chave: Lean Manufacturing, implementação de Lean, melhoria contínua, treinamento Lean, liderança, compromisso de endereço

JEL: L23, O33 


\section{INTRODUCCIÓN}

Colombia inició de forma tardía la implementación de herramientas de manufactura esbelta, al interior de las empresas. De acuerdo con Arrieta, Botero, y Romano (2010:143), en Colombia solo se registran iniciativas de implementación Lean a finales del siglo XX e inicios del siglo XXI, en empresas multinacionales que tienen operaciones en el país como General Motors-Colmotores, Tetra Pak, Unilever Andina y Siemens; que ya sea por disposición central o por iniciativa propia, realizaron la implementación de algunas de las herramientas de esta filosofía. Lean Manufacturing, que inicialmente se conoció como el sistema de producción Toyota (TPS, por sus siglas en Inglés), fue implementado en Japón durante la mitad del siglo XX y a nivel mundial se inició su conocimiento e implementación a partir de 1970, buscando incrementar el desempeño empresarial mediante el desarrollo de modelos de gestión que permitieran el mejoramiento continuo y flexible de las formas de producción (Pérez, 2011: 175).

En Antioquia, Sofasa fue una de las líderes en su implementación, gracias a la integración como accionista en 1989 de “Toyota Motor”. En 1991, la ensambladora de vehículos, inicia la implantación de herramientas Lean, aprovechando la experiencia de la fábrica en Japón, mientras que otras empresas industriales antioqueñas pertenecientes al GEA (Grupo Empresarial Antioqueño) también le siguieron los pasos (Arrieta et al., 2010: 144).

La filosofía Lean se basa en la premisa de hacer más con menos: menos tiempo, menos espacio, menos esfuerzos humanos, menos maquinaria, menos materiales, siempre cumpliendo lo que los clientes quieren, cuando lo quieran, donde lo quieran, a un precio competitivo, en las cantidades y variedades que ellos quieran, y siempre de excelente calidad (Alukal \& Manos, 2006:2; Villaseñor \& Galindo, 2007:28). Para poder lograr estas premisas, la filosofía Lean ofrece más de 20 herramientas; que para ser implementadas con éxito, dependen de consultorías, entrenamiento y especialmente el compromiso de la gerencia. En el proceso de implementación Lean en empresas Colombianas, algunas de las iniciativas han sido exitosas, mientras que para otras organizaciones se han presentado dificultades, obteniendo resultados negativos o no cumpliendo con las expectativas previstas.

En el presente documento se pretende encontrar cuales han sido esos factores claves del éxito en la implementación de la filosofía Lean y/o de algunas de sus herramientas, mediante una investigación de estudios de caso, en empresas con sede en Colombia.

Si bien la filosofía de Lean Manufacturing ha logrado sobresalir como componente esencial del mejoramiento continuo por más de 50 años, son pocos los casos de aplicación que han sido ejecutados y documentados en Colombia. De acuerdo con Valencia \& Plazas (2010:8-10) en cierto modo, las herramientas que han sido exitosas en las compañías japonesas pueden enfrentarse a algunas barreras en las compañías colombianas, entre las cuales se encuentran la gestión de los sistemas de información, relaciones cliente-proveedor, cultura organizacional, estabilidad 
laboral y rotación de personal. Más aun, dichos casos se limitan a algunos sectores industriales donde se destaca el automotriz y metalmecánico, al igual que el uso de algunas herramientas, siendo el SMED la más empleada con un 36\% (Arrieta Posada, Muñoz Domínguez, Salcedo Echeverri, \& Sossa Gutiérrez, 2011: 6-9).

Así pues, se han realizado estudios en compañías dedicadas a la lavandería, procesamiento de cítricos; encuadernación y sector gráfico (Cardona, 2013), donde se destaca la importancia del personal que participa en los proyectos de desarrollo de Lean como eje central de su implementación, incluyendo a la alta dirección, mandos medios, ingenieros y operarios.

También se encuentran documentados procesos de implementación de Lean Six Sigma (LSS). Uno en el sector alimenticio, en la elaboración de mezclas de nutrición parenteral (2014), y otro en la elaboración de muebles de madera (2014). Del mismo modo, se encuentra esta herramienta (LSS), aplicada en proyectos logísticos (Mantilla Celis \& Sánchez García, 2012), transporte en el sector minero (Arango Serna, Gil Gomez, \& Zapata Cortés, 2009) y en el sector textil/confección (Barón \& Rivera, 2014), para reducir tiempos muertos, sobrecostos y variabilidad en la cadena de suministro, teniendo en cuenta la estrategia, clientes, procesos, comunicación, optimización de rutas, proveedores, herramientas Lean y reducción de mudas.

El Lean también ha sido empleado en el sector servicios en organizaciones de sanidad u hospitalarias, que en 2014 documentaron con estudios de caso la aplicación de Lean en dos unidades de emergencia (Pública, privada), en las cuales se logró incrementar la generación de valor para los pacientes mediante reducción en los problemas de acceso a la información, eliminación de retrasos innecesarios y programación de turnos de personal.

Paralelamente, se han elaborado investigaciones basadas en revisiones bibliográficas que dan cuenta de los avances en el área y de los posibles beneficios que pueden obtener las compañías a partir de su implementación. De aquí, se genera un interés generalizado por un introducir el concepto de Lean como parte del Pensamiento Sistémico y los Proyectos Educativos Institucionales, como ocurrió en la Universidad Icesi (Dinas, Franco, \& Rivera, 2010), el objetivo es mejorar la comprensión de los estudiantes en cuanto al sistema Lean.

Tal como ocurre con el número de investigaciones en Lean que han sido documentadas en Colombia, en el Valle de Aburrá y Medellín son pocos los casos que yacen en los repositorios institucionales y bases de datos. Como ilustración, se elaboraron un par de estudios de caso para evaluar el nivel de implementación de herramientas Lean en pymes del Valle de Aburrá (Arrieta Posada, Botero Herrera, \& Romano Martinez, 2010) en el sector textil y (Pérez Rave, Patiño Rodríguez, \& Úsuga Manco, 2010) en el sector industrial. Entre los principales hallazgos, se encontró que las empresas no han desarrollado una cultura y una filosofía del Lean, y por ende, el uso de herramientas es reducido, siendo las menos implementadas el Intercambio Electrónico de Documentos o Información EDI, certificación de 
proveedores, Kanban, 5's, six sigma, SMED y TPM. Sin embargo, hay herramientas como el control estadístico de procesos y el benchmarking que han traído beneficios a las compañías.

También, en un estudio realizado por Pérez Rave y otros (2011: 404-406), en nueve Pymes manufactureras de la ciudad, mostró que la capacitación del personal en 5's, 7 desperdicios, gerencia visual y trabajo en equipo, permite reducir tiempos de espera, transporte, procesos y movimientos innecesarios en un $25,5 \%$ en promedio. Los principales conceptos Lean se abordan a continuación.

\section{LA FILOSOFÍA LEAN}

El Lean Manufacturing o manufactura esbelta, es un modelo de organización y gestión del sistema de fabricación -personas, materiales, máquinas y métodosque a través de la mejora continua persigue aumentar la calidad, el servicio y la eficiencia, mediante la identificación y eliminación del desperdicio; entendiendo como desperdicio todas aquellas actividades que no aportan valor al producto y por las cuales el cliente no está dispuesto a pagar (Madariaga, 2013: 12; Rajadell \& Sánchez, 2010: 19).

Esta filosofía nace a mediados del siglo XX gracias a tres miembros de la familia Toyoda (Sakichi, Kiichiro y Eiji Toyoda) y a Taiichi Ohno ingeniero mecánico de la compañía Toyota Motor, quien publicó en 1978 el libro "Toyota seisan hoshiki", traducido al inglés en 1988 con el título "Toyota Production System: Beyond Large-Scale Production” (Madariaga, 2013: 4), como resultado de la tarea puesta por Eiji Toyoda, quien quería mejorar el proceso de manufactura de Toyota hasta igualarlo con la productividad de Ford (Villaseñor \& Galindo, 2009: 19).

Algunos de los principios del Sistema de Producción Toyota fueron tomados de metodologías ya propuestas en los Estados Unidos como son: "el flujo continuo", presentado por Ford; el "Sistema Halar" usado en los supermercados norteamericanos y el "ciclo Deming" o ciclo Planear-Hacer-Revisar-Actuar (PHVA), que se volvió piedra angular del mejoramiento continuo (Villaseñor \& Galindo, 2009: 15). El TPS generó una nueva cultura industrial, conocida en occidente como pensamiento Lean, el cual quedó definitivamente acuñado en los 90's con el libro The machine that changed the world (La máquina que cambió al mundo), donde Womack, Jones y Roos expusieron de forma didáctica el nuevo paradigma de producción de las empresas automovilísticas japonesas (Alukal \& Manos, 2006: 4; Madariaga, 2013: 3-5).

De acuerdo con Monden (1996: 20), el Sistema de Producción de Toyota tiene como principales objetivos: obtener beneficios a través de la reducción de costos, eliminar el exceso de producción, controlar la cantidad, asegurar la calidad y respetar la dimensión humana. En la actualidad Lean se ha convertido en una estrategia especialmente importante en las empresas. Algunas de las razones claves son: permite competir efectivamente en una economía global, afronta la presión de los clientes en cuanto a la reducción de precios, permite una rápida adaptación a los cambios tecnológicos, ayuda en la adopción, con mayor facilidad, de estándares de calidad como la norma ISO 9001, y es definitivo a la hora de cumplir con las 
altas expectativas de los clientes y a estandarizar los procesos, entre otros aspectos (Alukal \& Manos, 2006: 6).

El Sistema Lean se compone de pilares, herramientas y técnicas que se encargan de sustentar los procesos que permiten alcanzar las metas y objetivos de mejora. Un resumen de estas herramientas se encuentra en la Tabla 1.

Tabla 1 - Principales herramientas del Lean Manufacturing

\begin{tabular}{|c|c|c|c|}
\hline $\begin{array}{c}\text { Tipo de } \\
\text { Herramienta } \\
\end{array}$ & Nombre & Descripción & Fuente \\
\hline \multirow{4}{*}{$\begin{array}{l}\text { Pilares del } \\
\text { Lean }\end{array}$} & Six Sigma & $\begin{array}{l}\text { Identificación y corrección de las causas de los } \\
\text { errores y al hacerlo reducir la tasa a un nivel de } 3.4 \\
\text { defectos por millón de oportunidades (DPMO) o } 6 \sigma \\
\text { - Liderazgo comprometido de arriba hacia abajo }\end{array}$ & $\begin{array}{l}\text { (Kwak \& Anbari, } \\
\text { 2006: 708-709); } \\
\text { (Arnheiter \& } \\
\text { Maleyeff, 2005: } \\
\text { 6-16) }\end{array}$ \\
\hline & $\begin{array}{l}\text { Justo a } \\
\text { Tiempo }\end{array}$ & $\begin{array}{l}\text { Sincroniza los proveedores y los procesos para } \\
\text { reducir buena parte del desperdicio, a partir del } \\
\text { flujo, calidad e intervención de los empleados. } \\
\text { - Reduce plazos de entrega, niveles de inventario, } \\
\text { mejora la calidad. } \\
\text { - Proporciona operaciones fluidas y retroalimen- } \\
\text { tación inmediata. } \\
\text { - Entregar al cliente lo que desea, en la cantidad } \\
\text { que desea y exactamente como lo desea. }\end{array}$ & $\begin{array}{l}\text { (Cuatrecas a s, } \\
\text { 2006); (Gaither \& } \\
\text { Frazier, 2000: 516- } \\
\text { 537) }\end{array}$ \\
\hline & JIDOKA & $\begin{array}{l}\text { Incorporación de sistemas y dispositivos que otor- } \\
\text { gan a las máquinas la capacidad de detectar que se } \\
\text { están produciendo errores. } \\
\text { - Detención manual o automática, del proceso de } \\
\text { producción, a partir de la detección de errores, } \\
\text { para prevenir despilfarros } \\
\text { - Automatización teniendo en cuenta al ser hu- } \\
\text { mano. } \\
\text { - Relación entre las personas y maquinaria a cargo. }\end{array}$ & $\begin{array}{l}\text { (Hernán dez \& } \\
\text { Vizán, 2013:55-58); } \\
\text { (Villaseñor \& Galin- } \\
\text { do, 2009: 72) }\end{array}$ \\
\hline & KAIZEN & $\begin{array}{l}\text { Cultura de mejora continua sostenible. } \\
\text { - Involucra a toda la estructura organizacional y } \\
\text { tiene costos relativamente bajos. } \\
\text { - Forma líderes para proponer mejoras en el largo } \\
\text { plazo. }\end{array}$ & $\begin{array}{l}\text { (Alukal \& Manos, } \\
\text { 2006:14-22); (Imai, } \\
\text { 2012: 1-14) }\end{array}$ \\
\hline $\begin{array}{l}\text { Herramientas } \\
\text { de } \\
\text { Seguimiento }\end{array}$ & $\begin{array}{l}\text { Gestión } \\
\text { Visual }\end{array}$ & $\begin{array}{l}\text { Conjunto de medidas de comunicación que plas- } \\
\text { man, de forma evidente y sencilla, la situación del } \\
\text { sistema productivo, especialmente en las anomalías } \\
\text { y despilfarros. } \\
\text { - Empodera y genera sentido de pertenencia en } \\
\text { los empleados. } \\
\text { - Demarca áreas, materiales, productos, equipos, } \\
\text { programas de producción. } \\
\text { - Emplea indicadores. }\end{array}$ & $\begin{array}{l}\text { (Alukal \& Manos, } \\
\text { 2006); (Hernández } \\
\text { \& Vizán, 2013: 52- } \\
\text { 54) }\end{array}$ \\
\hline
\end{tabular}




\begin{tabular}{|c|c|c|c|}
\hline $\begin{array}{c}\text { Tipo de } \\
\text { Herramienta }\end{array}$ & Nombre & Descripción & Fuente \\
\hline \multirow{6}{*}{$\begin{array}{c}\text { Herramientas } \\
\text { Operativas }\end{array}$} & $5 S^{\prime} s$ & $\begin{array}{l}\text { Conformado por: Seiri (Clasificación), Seiton (Or- } \\
\text { den), Seiso (Limpieza), Seiketsu (Estandarización); } \\
\text { Shitsuke (Disciplina). } \\
\text { - Evita problemas derivados del desorden y la falta } \\
\text { de instrucciones. } \\
\text { - Proporciona bienestar, disciplina y un ambiente } \\
\text { armónico. }\end{array}$ & $\begin{array}{l}\text { (Villaseñor \& Galin- } \\
\text { do, 2009:79); (Ra- } \\
\text { jadell \& Sánchez, } \\
\text { 2010: 48-66) }\end{array}$ \\
\hline & $\begin{array}{l}\text { SMED Single- } \\
\text { Minute } \\
\text { Exchange of } \\
\text { Dies }\end{array}$ & $\begin{array}{l}\text { Conjunto de técnicas que persiguen la reducción de } \\
\text { los tiempos de preparación de máquina } \\
\text { - Estandarización mediante la instalación de nue- } \\
\text { vos mecanismos, plantillas y anclajes funcionales, } \\
\text { elimina ajustes tiempos muertos. }\end{array}$ & $\begin{array}{l}\text { (Villa señ or \& } \\
\text { Galindo, 2009:61- } \\
\text { 62); (Hernández \& } \\
\text { Vizán, 2013: 42) }\end{array}$ \\
\hline & $\begin{array}{l}\text { TPM - Total } \\
\text { Productive } \\
\text { Maintenance }\end{array}$ & $\begin{array}{l}\text { Conjunto de técnicas orientadas a eliminar las } \\
\text { averías a través de la participación y motivación de } \\
\text { todos los empleados. } \\
\text { - Previene pérdidas en todas las operaciones de } \\
\text { la empresa. } \\
\text { - Maximiza la efectividad y alarga la vida del } \\
\text { equipo. }\end{array}$ & $\begin{array}{l}\text { (Villa señor \& } \\
\text { Galindo, 2009:66); } \\
\text { (Hernández \& } \\
\text { Vizán, 2013: 48-52) }\end{array}$ \\
\hline & Kanban & $\begin{array}{l}\text { Sistema de control y programación sincronizada } \\
\text { de la producción basado en tarjetas, que comunica } \\
\text { información sobre el flujo del producto. }\end{array}$ & $\begin{array}{l}\text { (Monden, 1996: } \\
\text { 26-30) }\end{array}$ \\
\hline & $\begin{array}{l}\text { Células de } \\
\text { manufactura }\end{array}$ & $\begin{array}{l}\text { Celdas de trabajo que se diseña para producir una } \\
\text { familia de partes o una cantidad limitada de familias } \\
\text { de partes, permitiendo un flujo continuo trans- } \\
\text { formando varios procesos, que trabajan de forma } \\
\text { independiente, en una celda de trabajo conjunta. } \\
\text { - Mejora la comunicación y utilización de personas } \\
\text { y equipos. } \\
\text { - Considera la formación, disposición y secuencia } \\
\text { de las máquinas. }\end{array}$ & $\begin{array}{l}\text { ( Villa s e ñ or \& } \\
\text { Galindo, 2009:55), } \\
\text { (Chang, Wu, \& Wu, } \\
\text { 2013: 439-449) }\end{array}$ \\
\hline & Poka-Yoke & $\begin{array}{l}\text { Instalación de dispositivos para detectar errores, } \\
\text { parar la producción y alertar al operario } \\
\text { - A prueba de errores, respetando la inteligencia } \\
\text { de los trabajadores. } \\
\text { - Prevenir la producción de defectos a través de la } \\
\text { detección temprana de errores. }\end{array}$ & $\begin{array}{l}\text { (Villaseñor \& Galin- } \\
\text { do, 2009: 83-85); } \\
\text { (Hernán de z \& } \\
\text { Vizán, 2013: 55-58) }\end{array}$ \\
\hline $\begin{array}{c}\text { Herramientas } \\
\text { de } \\
\text { Diagnóstico }\end{array}$ & VSM & $\begin{array}{l}\text { Mapa en el que se especifica la cadena de valor de } \\
\text { la organización tanto en áreas productivas como } \\
\text { gerenciales. } \\
\text { - Identifica el flujo de procesos y los desperdicios. } \\
\text { - Da respuesta a problemáticas de comunicación, } \\
\text { personal, material, equipos y procesos }\end{array}$ & $\begin{array}{l}\text { (Sullivan, McDon- } \\
\text { ald, \& Van Aken, } \\
\text { 2002); (Nash \& Pol- } \\
\text { ing, 2008: 9-201) }\end{array}$ \\
\hline
\end{tabular}


La herramienta Six Sigma inicialmente no formaba parte de las herramientas de Toyota, esta fue desarrollada por Motorola, pero gracias a los ambiciosos estándares de calidad y de la disciplina exigida a los gerentes y líderes, se incluyó dentro de las herramientas Lean.

\section{METODOLOGÍA}

La metodología empleada es de carácter cualitativo, con un alcance exploratorio descriptivo, basada en estudios de caso, de cuatro organizaciones colombianas ubicadas en el Valle de Aburrá y una cerca de la capital del país, las cuales han implementado herramientas de la filosofía Lean Manufacturing.

El muestreo realizado es no probabilístico por conveniencia. La selección se realizó a partir de una lista de 52 empresas cuyo nombre había sido mencionado por revistas especializadas, basada en investigaciones previas sobre implementación de herramientas Lean, con sede en Colombia. Inicialmente se hizo un contacto telefónico con los responsables de los procesos, de implementación, en el cual se les informó el interés de la investigación y posteriormente, vía telefónica o correo electrónico se concertó la visita para la entrevista. Los entrevistados son los responsables directos del proceso de implementación de herramientas Lean, quienes conocen de primera mano las ventajas y beneficios percibidos así como los tropiezos y dificultades presentadas. En la visita a las empresas, una vez concluida la entrevista, los investigadores tuvieron la oportunidad de hacer un recorrido por las áreas donde se implementaron procesos y herramientas Lean y se pudieron hacer observaciones de su aplicación. La recolección de la información se llevó a cabo durante los meses Junio a Septiembre de 2015.

La información primaria se obtuvo a partir del diseño de un formulario que sirvió de base para las entrevistas personales en profundidad, con los líderes de proceso de dichas compañías. El formulario contiene cinco secciones. La primera aborda las generalidades de las metodologías de gestión de la empresa, definición de Lean Manufacturing, herramientas, fases de ejecución y beneficios. La segunda y tercera sección contienen los componentes motivacionales, organizacionales y de seguimiento que permitieron la implementación del Lean Manufacturing. En la cuarta sección se busca obtener información acerca de las características y requerimientos previos a la adopción del Lean; y la última, busca documentar las barreras y dificultades que se presentaron durante la implementación de estas herramientas y filosofía. El formulario base para las entrevistas en profundidad, podrá ser consultado haciendo click aquí ${ }^{\text {. }}$

Las organizaciones que participaron en la investigación son Incolmotos Yamaha S.A., Siemens S.A., Único Interior S.A.S. y dos Compañías, una del sector Textil y otra del sector de Electrodomésticos, de quienes nos reservamos el nombre por petición explicita de sus directivos.

1. La dirección Web donde se encuentra la guia de las entrevistas en profundidad es: https://onedrive.live. com/redir?resid=22D2E481EF68B9A5!5075\&authkey=!AAL_fPMP3vCUUcY\&ithint=file\%2cpdf 
También se obtuvo información a partir de fuentes secundarias mediante la revisión detallada de repositorios institucionales, bases de datos, archivos, informes y revistas especializadas en el área.

Con la información recabada, se procedió a tabular datos, y se enumeraron los hallazgos más importantes y coincidentes en la implementación exitosa de algunas herramientas.

Entre las limitaciones encontradas en el proceso de investigación, algunas de las empresas con liderazgo en la implementación Lean, guardan recelo en compartir el conocimiento y los adelantos efectuados, lo que dificultó la concertación para la entrevista. Sus líderes o los directivos principales, al conocer del objetivo general los "factores claves de éxito", respetuosamente declinaron hacer parte de la investigación.

\section{RESULTADOS Y DISCUSIÓN}

La investigación determinó que hay cuatro factores claves en el éxito de la aplicación de las filosofías Lean, como se aprecia en la Gráfica 1. i) el compromiso de la alta dirección (gerencias, presidencias, vicepresidencias y direcciones de áreas), ii) el seguimiento continuo, con indicadores de gestión apropiados, iii) el liderazgo y iv) el entrenamiento. Estos factores serán discutidos a continuación:

Figura 1 - Factores claves en la aplicación de filosofías Lean

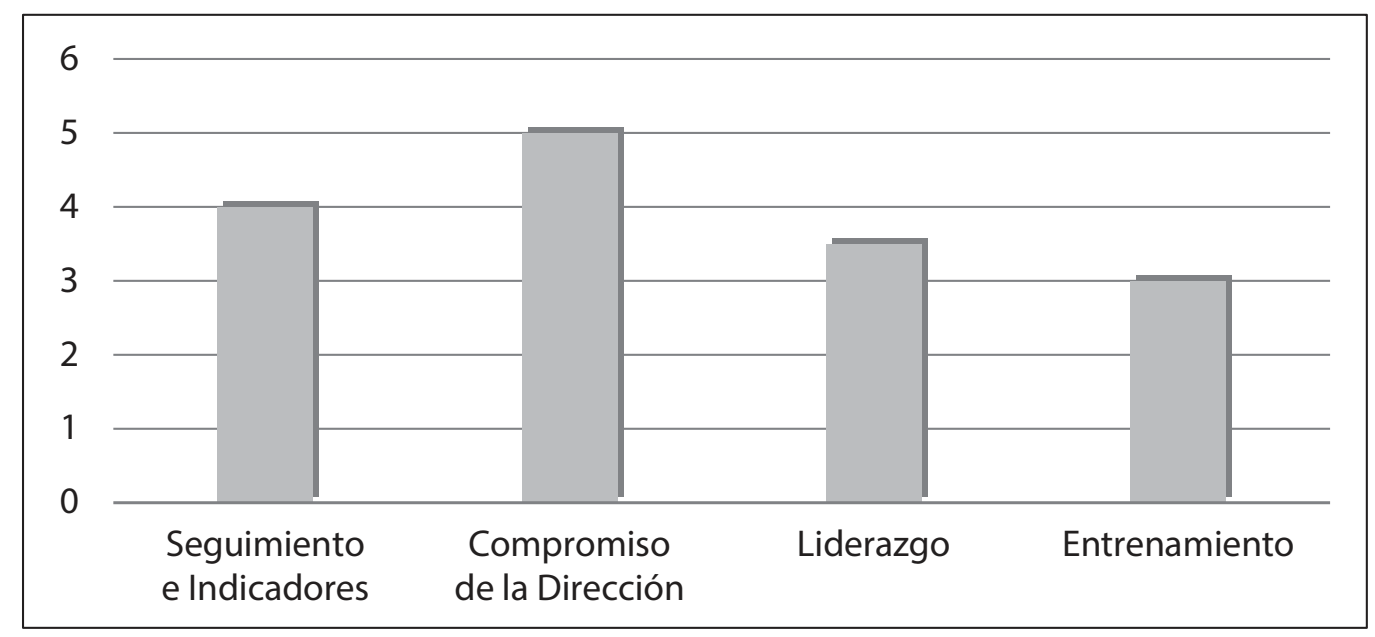

Fuente: Elaboración propia de los autores

\subsection{Compromiso de la Dirección}

En 3 de las empresas seleccionadas, las directrices generales vienen de las sedes principales localizadas en países externos, pero precisamente esos directivos entienden la magnitud de la implementación y los grandes beneficios que esta trae a la empresa, por tanto hicieron todo lo posible por dejar que los líderes se empoderaran y entregaran el resultado esperado. De igual manera sucedió con las empresas nacionales, aunque inicialmente sus directivos no entendían cuál era la 
magnitud de la implantación, realizaron las inversiones, aprobaron los cambios en la organización y ofrecieron apoyo irrestricto a los líderes Lean, directores de producción y directivos involucrados, para alcanzar los objetivos propuestos.

En un modelo, propuesto por Cardona (2013: 137) para la implementación de Herramientas Lean en empresas editoriales, este resalta la importancia de que toda información fluya a partir de los directivos, lo que permite a los empleados aceptar más fácilmente las nuevas directrices y la aversión al cambio sea menor. Se encontró también un proyecto de implementación Lean con Six Sigma en la elaboración de muebles de madera, en la cual dentro de los hallazgos se resalta que a pesar de generar impactos positivos a nivel financiero y operacional, la evaluación de los resultados del portafolio de proyectos aún es incipiente y que dentro de los cambios fundamentales necesarios para el éxito se recomienda un mayor compromiso de la dirección que permita profundizar el cambio en la cultura organizacional, el enfoque al cliente, y se incluyan temas necesarios como la implementación de indicadores claves, formación, capacidades, competencias, nivel de conocimiento en metodologías y herramientas Lean, entre otros.

\subsection{Liderazgo}

De las empresas entrevistadas en Colombia, todas delegaron en líderes con conocimiento y entrenamiento la implementación del proyecto. Algunas de ellas contaron adicionalmente con empresas consultoras que sirven de guía y evaluación en las áreas y herramientas objeto de la implantación.

La ausencia de líderes, o líderes con poca experiencia en la dirección de proyectos, obstaculizan el éxito de la implementación. Un investigación similar realizada por Achanga, Shehab, Roy y Nelder de la universidad de Cranfield en PYMES del Reino Unido, encontró que en muchas la implementación había estado a cargo de los dueños y gerentes (que en muchos casos son los mismos), "pero estos no contaban con el Know-How necesario para cerrar con éxito el proyecto Lean" (2006: 467). Sin embargo también encontraron que hoy, en la mayoría de empresas, existe un interés muy alto por iniciativas de mejora estratégica, generando así comportamientos de liderazgo. Sin embargo los líderes actuales se centran en resolver las pequeñas crisis empresariales del día a día, mientras que el enfoque en la implementación de herramientas Lean, que les dejaría una base más firme para el éxito reduciendo costos y haciendo un uso racional de los recursos, es pospuesta, esperando encontrar tiempos mejores para hacerlo (Achanga et al., 2006).

\subsection{Seguimiento e Indicadores}

Todas las organizaciones entrevistadas estuvieron de acuerdo en que si los indicadores no se atendían a tiempo, se causaban enormes retrasos a la implantación Lean, independiente de la herramienta que se estuviera utilizando. Adicionalmente y en concordancia con el ciclo planteado, también estuvieron de acuerdo en que el direccionamiento estratégico y la planeación anticipada de todas las áreas es vital para poder poner en marcha las herramientas Lean. Es de gran relevancia conocer, por ejemplo, desde el año anterior, cuales van a ser los niveles de venta esperados para cada uno de los meses del siguiente, en cuales meses se van a realizar campañas especiales y cuando se hará la introducción de nuevos productos 
al mercado, de tal suerte que todas las áreas de producción mercadeo y ventas, se encuentren preparadas con antelación a las fechas publicadas.

En la empresa Unico, uno de los líderes confirmaba que una pequeña desviación de uno de los Indicadores del SMED afectaba inmediatamente todo el proceso de producción, el cual a la vez se veía reflejado en incremento de costos, y afectaciones negativas en el área comercial por incumplimientos y retrasos en las entregas.

Como se mencionó de estudios realizados en 2014, para que el portafolio de proyectos pudiera generar los resultados esperados era imprescindible la implementación de indicadores claves que permitieran "eliminar la subjetividad y poder profundizar en la mejora continua y la excelencia operacional”.

Los indicadores de gestión son una de las herramientas más poderosas para el cambio organizacional, estos son un ingrediente fundamental en el seguimiento y éxito de los proyectos. Esta es una disciplina que permite alinear el desempeño con la estrategia (Eckerson, 2009: 4).

Los indicadores claves de gestión (KPI’s) -por sus siglas en inglés-, miden la salud de la empresa y aseguran que todos los individuos en todos los niveles "vayan a la par " persiguiendo los mismos objetivos y estrategias (Bauer, 2004). También proporcionan el punto focal de coordinación, colaboración y normalización de toda la empresa.

Aunque los indicadores pueden ser un arma de doble filo, puesto que si estos están construidos equivocadamente, podrían generar consecuencias inesperadas que van desde la desmoralización de los empleados hasta el fracaso completo de procesos empresariales, también los hay bien fundamentados en un ciclo de 4 fases, a partir del direccionamiento estratégico en el cual se plasman las estrategias y de estas surge la misión, valores, metas, incentivos y mapas estratégicos. Con esta información se realiza la planeación, se elaboran los presupuestos y se fijan los objetivos y métricas. Como tercer paso se diseñan los cuadros de reporte de indicadores, el seguimiento y control y las herramientas de análisis, y por último se plantean las acciones y ajustes requeridos en caso de las deviaciones e imprevistos (Eckerson, 2009: 6-12).

\subsection{Entrenamiento}

El entrenamiento es parte esencial para poder llevar con éxito la implementación de las Herramientas y Filosofías Lean, sin embargo este entrenamiento tiene unas características especiales y únicas que lo diferencian de un entrenamiento estándar, por lo tanto se dedicará especial atención a los detalles de este ítem.

Para encontrar el éxito en la implementación, se deberá tomar el Modelo de Producción Toyota (TPS),en donde todo es diferente: El estilo de gerenciamiento, el estilo de liderazgo, los indicadores de gestión, la estructura organizacional y la cultura (Piatkowski, 2004: 2). 
Uno de los grandes entrenadores de Lean, en Canadá, Marek Piatkowski dice que en la capacitación y entrenamiento se deben tener en cuenta estos cinco importantes aspectos:

\subsubsection{El entrenamiento debe ser llevado a cabo por los gerentes y por los líderes Lean}

El entrenamiento Lean es muy similar a la trasferencia de tradiciones en una familia, es decir, el líder o el Gerente deberán enseñar a hacer, a todos sus subalternos. El ejemplo en este caso, es esencial.

En una de las empresas entrevistadas, solo cuando se puso en práctica el entrenamiento de tres fases se logró pasar al siguiente nivel. Este entrenamiento ellos lo llaman así porque consta de "Digo y Hago" (por el entrenador o líder, para que el aprendiz pueda interiorizar el entrenamiento), Hago y Digo (por el aprendiz, para validar lo aprendido), Valido y Hago (Por el Aprendiz, teniendo el visto bueno del supervisor o líder).

\subsubsection{El entrenamiento se hace en el campo de trabajo}

Todos los gerentes y líderes Lean deberán saber hacer lo que van a enseñar. No se puede enseñar desde la teoría. El entrenamiento se debe hacer en la planta. Posiblemente se destine alguna línea de producción o algún lugar dentro de la planta para practicar lo aprendido, pero el conocimiento práctico es esencial y el trabajo real es parte del entrenamiento.

En todas las empresas entrevistadas se tienen planes de entrenamiento y evaluación, de los procedimientos, y de acuerdo con los ajustes y mejoras, la realización de re-entrenamiento que mantenga a todos los operarios y empleados actualizados.

\subsubsection{Se deben entender los principios del Sistema de Producción Toyota TPS}

Es esencial que los conocimientos teóricos y bases del sistema, sean conocidos por todos y cada uno de los empleados y se practiquen a diario con: Mejora continua, Kanban, Mantenimiento, condiciones competitivas, y principios y políticas de: calidad, seguridad, atención, y relaciones laborales.

Las empresas entrevistadas tienen líderes que han sido formados, en las plantas, en Universidades locales que cuentan con programas de Lean Manufacturing y por firmas consultoras externas que tienen personal certificado en la implantación de estas herramientas. En algunas de las empresas los líderes han sido también entrenados en fábricas de otros países ya sea por planes de intercambio o por entrenamiento especializado.

\subsubsection{Los líderes deben desarrollar conocimientos y competencias Lean}

El conocimiento de los roles y responsabilidades en la organización, y de los elementos de trabajo; y competencias como entrenador, como líder y en la mejora continua son vitales en cada uno de los líderes.

Estos deben estar entrenados para convertirse en practicantes Lean, capaces de enseñar, guiar y ser mentores en la implementación del Lean. Los profesionales Lean (gerentes y supervisores) deberán transmitir, no sólo el conocimiento de herra- 
mientas y metodologías Lean, sino también sus roles y responsabilidades. También deben saber administrar en un entorno Lean, aplicar este conocimiento todos los días, y hacerse responsables del éxito o fracaso del proceso de implementación.

Muchas veces la responsabilidad, en Colombia, se delega al entrenador externo o a la firma consultora o a un departamento de calidad sin un soporte adecuado y la capacitación pertinente.

\subsubsection{Desarrollo y crecimiento de Gerentes y líderes}

El sistema Lean deberá auspiciar el incremento en conocimientos y habilidades de liderazgo de sus líderes, para que el crecimiento y mejoramiento sea continuo, desarrollando habilidades especiales que les permitan fácilmente aplicar estandarizaciones, coordinar procesos que permitan el Justo a Tiempo, y desarrollar técnicas que permitan halar todo el tiempo (Piatkowski, 2004).

El total de las empresas entrevistadas contaron con fuertes planes de entrenamiento; sin embargo para algunas de las empresas fue más difícil lograr la estandarización de la aplicación de las herramientas por factores culturales que tuvieron que ser tenidos en cuenta para tropicalizar o adaptar el Lean, tales como la implementación de incentivos o demostraciones prácticas de que los jefes y líderes si saben hacer.

Lo anterior se pudo corroborar en una de las empresas entrevistadas, la cual contrató con una firma consultora extranjera todo el proceso de capacitación y entrenamiento a sus líderes y operarios. Transcurridos dos años, la firma consultora certificó el nivel de entrenamiento y se marchó. 6 meses después, los procesos de fabricación tenían más problemas que antes de la capacitación. Se detectó que los consultores solo se habían limitado a enseñar unas técnicas, pero la cultura organizacional, los problemas de identidad cultural, el direccionamiento de la organización y sus metas no formaban parte de esos entrenadores, por lo tanto la tarea quedó incompleta. Los líderes y entrenadores de campo deben pertenecer a la organización. Una vez se hicieron los correctivos, todos los procesos de producción pudieron ser alineados a la filosofía y gracias a los beneficios obtenidos, cuentan con certificación internacional y maquilan para marcas de renombre mundial.

\section{OTROS HALLAZGOS}

\subsection{Capacidad Financiera}

Aunque ninguna de las empresas cuantificó con exactitud el monto invertido en cada uno de los ítems de la implementación (Consultoría, Entrenamiento, Infraestructura, Máquinas y Herramientas, entre otros), es claro que esta, así sea de una sola herramienta, conlleva el egreso de rubros cuyo retorno de inversión no es fácilmente cuantificable y es de largo plazo. En las culturas occidentales es muy común pedir resultados inmediatistas y si estos no están a la vista, muchos de los empresarios prefieren volver a la forma tradicional de hacer las cosas. La visión de largo plazo en los directivos y líderes es esencial para que se vean los frutos. 


\subsection{Desarrollo de proveedores}

Como parte de la madurez del proceso de implementación de filosofías Lean, estas empresas decidieron extender las capacitaciones y metodologías a los proveedores más importantes de sus organizaciones, para que aspectos como la calidad, tiempos de entrega, documentación, empaque y otros aspectos claves en la cadena de suministro cumplan los estándares propuestos al interior de la empresa.

La iniciativa ha sido muy bien recibida y con algunas de estas empresas ya hay inclusive integraciones In House.

\subsection{Herramientas Implementadas}

Cada empresa, y de acuerdo con su direccionamiento y necesidades eligió la implementación de ciertas herramientas, algunas con la aplicación general en todos los procesos de la empresa y otras solo en las áreas de producción. La herramienta más generalizada es la de las 5S's, siendo seguida por Celdas de Manufactura y Gestión Visual como se puede apreciar en la Tabla 2.

Tabla 2 - Herramientas Lean más utilizadas por la empresas

\begin{tabular}{|c|c|c|c|c|c|c|}
\hline $\begin{array}{l}\text { Empresa/ } \\
\text { Herramienta }\end{array}$ & $\begin{array}{l}\text { Siemens } \\
\text { S.A. }\end{array}$ & $\begin{array}{l}\text { Único } \\
\text { Interior } \\
\text { S.A.S. }\end{array}$ & $\begin{array}{c}\text { Empresa } \\
\text { textil }\end{array}$ & $\begin{array}{l}\text { Empresa de } \\
\text { electrodo- } \\
\text { mésticos }\end{array}$ & $\begin{array}{l}\text { Incolmotos } \\
\text { Yamaha S.A. }\end{array}$ & $\begin{array}{l}\text { Porcentaje } \\
\text { aplicación }\end{array}$ \\
\hline $5^{\prime} S$ & $x$ & $x$ & $x$ & $X$ & $x$ & $100 \%$ \\
\hline $\begin{array}{c}\text { Celdas de } \\
\text { Manufactura }\end{array}$ & $x$ & $X$ & & $X$ & $X$ & $80 \%$ \\
\hline $\begin{array}{c}\text { Gestión } \\
\text { Visual }\end{array}$ & $X$ & $X$ & & $X$ & $X$ & $80 \%$ \\
\hline TPM & $x$ & $x$ & & $X$ & $x$ & $80 \%$ \\
\hline Six Sigma & $X$ & & & $X$ & $X$ & $60 \%$ \\
\hline SMED & $x$ & $x$ & & & $x$ & $60 \%$ \\
\hline VSM & & $x$ & & $X$ & $X$ & $60 \%$ \\
\hline Kanban & & & & $X$ & $x$ & $40 \%$ \\
\hline Kaizen & $x$ & & & $X$ & $X$ & $40 \%$ \\
\hline JIT & $x$ & $x$ & & & & $40 \%$ \\
\hline $\begin{array}{l}\text { Producción } \\
\text { Sincrónica }\end{array}$ & & & & & $X$ & $20 \%$ \\
\hline
\end{tabular}

Fuente: Elaboración propia de los autores.

Otras herramientas que son muy sensibles en la cadena de producción como Poka Yoke o Jidoka, se usan a discreción, puesto que la inversión en la implementación y la parada completa de toda una línea de producción genera costos elevados cuyo retorno aun no es fácilmente cuantificable. 


\section{CONCLUSIONES}

Sin duda alguna para el éxito de la implementación de la filosofía Lean, en empresas colombianas, es crítico y definitivo el compromiso de dueños y altos directivos de las organizaciones, quienes deben Aprender a Hacer y a la vez Dejar Hacer. Si por el contrario son pequeñas y medianas empresas, el dueño o Gerente se deberá asesorar de expertos en Lean, que lo entrenen y a la vez entrenen a líderes y supervisores en los aspectos claves del Lean y sean estos últimos quienes lleven a cabo la capacitación y entrenamiento a la planta de operarios y empleados en general.

Un Buen liderazgo asegura e impulsa la creación o mejora de habilidades en los grupos de trabajo, permitiendo que la implementación se enriquezca con las herramientas disponibles, se generen ideas innovadoras y se integren nuevas tecnologías al proceso. Por esta razón en la implementación Lean se deberá propender por la formación y desarrollo de esta competencia en supervisores y mandos medios.

La formulación de Indicadores de gestión Lean, el seguimiento y la puesta en marcha de acciones de mejora a partir de estos, son vitales para poder medir la eficiencia y eficacia, no solo del proceso de implantación sino de las herramientas que se vayan implementando.

El entrenamiento es tal vez uno de los factores más costosos y lentos de llevar a cabo, por lo tanto debe ser a la medida, es decir, se deben monitorear y tener en cuenta los aspectos culturales propios de cada empresa y región, adaptando o incluyendo factores que sean relevantes para el logro de los objetivos en capacitación, entrenamiento e implantación de la filosofía.

Puesto que la sensibilización, capacitación, entrenamiento e implementación de filosofías Lean tienen un costo elevado, es recomendable que los dueños y directivos principales se integren a este proceso y además provean no solo los recursos sino el respaldo a los líderes y gerentes del proyecto, destinando recursos, tiempo y entrenamiento, necesario para la ejecución y conviertan esta filosofía en su visión y direccionamiento estratégico personal y empresarial.

Cuando se contraten firmas externas para la capacitación y entrenamiento, la empresa se debe asegurar que los consultores y asesores entienden la dirección de la empresa, sus metas y objetivos, pero también las restricciones de recursos para el Lean. Deberán a su vez hacer equipo con los líderes Lean, propios de la empresa, para asegurarse que la responsabilidad por la ejecución de las iniciativas Lean sean desarrolladas por los empleados una vez finalice el entrenamiento.

En el medio colombiano se podrían realizar nuevas investigaciones, enfocadas a la pequeña y mediana empresa en las cuales se pudieran entrevistar a supervisores y operarios para documentar tanto las barreras, como los factores claves de éxito, ampliando además la cobertura a otros sectores geográficos del país. 


\section{REFERENCIAS}

1. ACHANGA, P.; SHEHAB, E.; ROY, R. \& NELDER, G. (2006). Critical success factors for lean implementation within SMEs. Journal of Manufacturing Technology Management, 17(4), 460-471. http://doi.org/10.1108/17410380610662889

2. ALUKAL, G. \& MANOS, A. (2006). Lean Kaizen: A Simplified Approach to Process Improvements (A. Hytinen \& P. 0'Mara, Eds.). Milwaukee, USA: William A. Tony. Retrieved from http://books.google.com/books?id=aohFNzEOBWkC\&pgis=1

3. ARANGO SERNA, M. D.; GIL GOMEZ, H. \& ZAPATA CORTÉS, J. A. (2009). Logística Esbelta Aplicada Al Transporte En El Sector Minero. Boletín de Ciencias de La Tierra (25), 121-136. Retrieved from http://agris.fa0.org/agris-search/search/display.do?f=2011/C0/C01005.xml;C02010G00060

4. ARRIETA POSADA, J.G.; BOTERO HERRERA, V. E. \& ROMANO MARTÍNEZ, M. J. (2010). Benchmarking about lean manufacturing in the textile sector in Medellin/ Benchmarking sobre Manufactura Esbelta (lean manufacturing) en el sector de la confeccion en la ciudad de Medellin, Colombia. Journal of Economics, Finance and Administrative Science, 15(28), 141-170.

5. ARRIETA POSADA, J. G.; MUÑOZ DOMINGUEZ, J. D.; SALCEDO ECHEVERRI, A. \& SOSSA GUTIÉRREZ, S. (2011). Aplicación Lean Manufacturing En La Industria Colombiana. Ninth LACCEI Latin American and Caribbean Conference (LACCEI'2011), Engineering for a Smart Planet, Innovation, Information Technology and Computational Tools for Sustainable Development, August 3-5, 2011, Medellin, Colombia, 1-11.

6. ARRIETA, J. G.; BOTERO, V. E. \& ROMANO, M. J. (2010). Benchmarking sobre manufactura esbelta (lean manufacturing) en el sector de la confección en la ciudad de Medellín, Colombia. Journal of Economics, Finance and Administrative Science, 31.

7. BARÓN, D. I. M. \& RIVERA, L. C. (2014). Cómo una microempresa logró un desarrollo de productos ágil y generador de valor empleando Lean. Estudios Gerenciales, 30(130), 40-47. http://doi.org/10.1016/j.estger.2014.02.007

8. BAUER, K. (2004). The Metrics That Drive Performance Management. Retrieved November 20, 2015, from http://search.proquest.com/openview/ dc6ab764896a552c4a38fc7dca106792/1?

9. CARDONA, J. J. (2013). Modelo para la implementación de técnicas lean manufacturing en empresas editoriales.

10. DINAS, J. A.; Franco, P. \& Rivera, L. (2010). Aplicación de herramientas de pensamiento sistémico para el aprendizaje de Lean Manufacturing. Sistemas y Telemática, 7(14), 109-144.

11. ECKERSON, W. W. (2009). Performance Management Strategies. Rento, WA. Retrieved from http://www.microstrategy.com/Strategy/media/ downloads/white-papers/TDWI_Performance-Management-Strategies.pdf

12. MADARIAGA, F. (2013). Lean manufacturing: Exposición adaptada a la fabricación repetitiva de familias de productos mediante procesos directos. Bubok Publishing S.L. Retrieved from https://books.google.com/books?id=mBgDGYRQZXMC\&pgis=1

13. MANTILLA CELIS, 0. L. \& SÁNCHEZ GARCÍA, J. M. (2012). Modelo tecnológico para el desarrollo de proyectos logísticos usando Lean Six Sigma. Estudios Gerenciales, 28, 23-43. http://doi.org/http://dx.doi.org/10.1016/50123-5923(12)70214-0

14. MONDEN, Y. (1996). El just in time hoy en Toyota (2a ed.). Bilbao: Ediciones Deusto S.A.

15. PÉREZ RAVE, J.; LA ROTTA, D.; SÁNCHEZ, K.;MADERA, Y.; RESTREPO, G.; RODRÍGUEZ, M.; PARRA, C. (2011). Identificación y caracterización de mudas de transporte, procesos, movimientos y tiempos de espera en nueve pymes manufactureras incorporando la perspectiva del nivel operativo. Ingeniare. Revista Chilena de Ingeniería, 19(3), 396-408. http://doi.org/10.4067/S0718-33052011000300009

16. PÉREZ RAVE, J.; PATIÑO RODRÍGUEZ, C. \& ÚSUGA MANC0, 0. (2010). Uso de herramientas de mejoramiento y su incidencia en costos, fallas y factores de éxito de grandes y medianas empresas industriales del Valle de Aburrá. Gestão \& Produção, 17(3), 589-602. http://doi.org/10.1590/ S0104-530X2010000300012

17. PÉREZ, J. (2011). El avión de la muda : herramienta de apoyo a la enseñanza-aprendizaje práctico de la manufactura esbelta Airplane of the muda: support tool for the teaching-learning experience of lean manufacturing. Revista Facultad Ingenieria Universidad de Antioquia, 58(58), 173-182.

18. PIATKOWSKI, M. (2004). Training Recommendations for Implementing Lean. Retrieved from http://www.lean.org/Search/Documents/492.pdf

19. RAJADELL, M. \& SÁNCHEZ, J. L. (2010). Lean Manufacturing. La evidencia de una necesidad. Madrid: Ediciones Díaz de Santos. Retrieved from http://books.google.com.co/books/about/Lean_Manufacturing_La_evidencia_de_una_n.html?id=IR2xgsdmdUoC\&pgis=1

20. VALENCIA, D. J. \& PLAZAS, J. A. (2010). Análisis de la implementación del lean manufacturing en las pymes Colombianas. In XVI International Conference on Industrial Engineering and Operations Management. Retrieved from http://www.abepro.org.br/biblioteca/enegep2010_ti_ st_119_776_17407.pdf

21. VILLASEÑOR, A. \& GALINDO, E. (2009). Manual de Lean Manufacturing. Guía básica. Editorial Limusa. 